\title{
Monitoring Dose Response of Enzyme Replacement Therapy in Feline Mucopolysaccharidosis Type VI by Tandem Mass Spectrometry
}

\author{
ALLISON CRAWLEY, STEVEN L. RAMSAY, SHARON BYERS, JOHN J. HOPWOOD, AND \\ PETER J. MEIKLE
}

Lysosomal Diseases Research Unit, Department of Genetic Medicine, Women's and Children's Hospital, North Adelaide, South Australia 5006, Australia. [A.C., S.L.R., S.B., J.J.H., P.J.M.], and Department of

Paediatrics, The University of Adelaide, Adelaide, South Australia 5000, Australia [S.B., J.H.H., P.J.M.]

\section{ABSTRACT}

\begin{abstract}
Mucopolysaccharidosis type VI is an inherited disorder of glycosaminoglycan metabolism characterized by organomegaly, corneal clouding, and skeletal dysplasia. Recent developments in the use of tandem mass spectrometry to measure sulfated monoand disaccharides have enabled us to perform noninvasive, biochemical monitoring during therapy regimes in mucopolysaccharidosis type VI cats in addition to established methods of disease evaluation. In this study, mucopolysaccharidosis type VI animals were given high-dose $(20 \mathrm{mg} / \mathrm{kg})$ enzyme replacement therapy for the first month after birth followed by low doses $(1 \mathrm{mg} / \mathrm{kg})$ for a further 2 mo and were compared with animals maintained on $1 \mathrm{mg} / \mathrm{kg}$ enzyme replacement therapy for $3 \mathrm{mo}$. A sulfated monosaccharide ( $N$-acetylhexosamine) and a sulfated disaccharide ( $N$-acetylhexosamine-uronic acid) were elevated in MPS VI cat urine and blood. These markers showed a clear discrimination between the treatment groups during the first 4 wk of therapy: values in the high-dose group were close to normal whereas those in the low-dose group were only slightly lower than the untreated mucopolysaccharidosis type VI cats. However, within 2 mo of cessation of the high-dose therapy there was minimal difference in the oligosaccharide levels, with both groups lying
\end{abstract}

between the untreated and unaffected cats. At the completion of the trial, subjective minor improvement was noted in overall physical disease features and also in lysosomal vacuolation in tissues from animals on the initial high-dose enzyme replacement therapy compared to the low-dose therapy animals. Initial highdose therapy reduced storage load in the animals but had no lasting clinical benefit over continuous low-dose therapy.

(Pediatr Res 55: 585-591, 2004)
Abbreviations
LSD, lysosomal storage disease
ERT, enzyme replacement therapy
GAG, glycosaminoglycan
ESI-MS/MS, electrospray ionization tandem mass
spectrometry
rf4S, recombinant feline 4-sulfatase
DMB, 1,9-dimethylmethylene blue
GIcNAc6S-d ${ }_{3}$, deuterated $N$-acetylglucosamine-6 sulfate
PMP, 1-phenyl-3-methyl-5-pyrazolone
HexNAc, $N$-acetylhexosamine
UA, uronic acid

LSD are a group of more than 45 distinct genetic disorders in which one or more lysosomal proteins necessary for the intracellular catabolism of compounds is absent or defective in function (1). Major clinical features of most of these disorders are neurologic deterioration and/or skeletal pathology. The overall incidence/prevalence of this group of disorders has been estimated to be 1 in 7700 births (2).

Received June 26, 2003; accepted November 21, 2003.

Correspondence: Peter Meikle, Ph.D., Department of Genetic Medicine, Women's and Children's Hospital, 72 King William Rd., North Adelaide, SA 5006, Australia; e-mail: peter.meikle@adelaide.edu.au

Supported by the National Health and Medical Research Council (Australia), TLH Research (U.S.A.), and the Wellcome Trust (U.K., Grant 060104Z/00/Z).

DOI: 10.1203/01.PDR.0000113789.30640.5C
The enzymes deficient in many of the LSD have been purified, characterized, cloned, and expressed. The resultant recombinant enzymes being used in ERT protocols. Fabry disease, Gaucher disease and mucopolysaccharidosis (MPS) type I are currently being treated by ERT (3-6). Clinical trials of ERT are underway for a number of other LSD, including MPS II, MPS VI, and Pompe disease $(5,7,8)$. Additional trials for MPS IVA and Niemann-Pick disease type B are proposed for the near future. Other therapies, including substrate deprivation and gene therapies, are either in clinical trial or actively being researched $(9,10)$. Extensive ERT trials have also been performed in a number of animal models of several different LSD, including the MPS I dog $(11,12)$, MPS I cat (13), MPS 
VI cat (14-16), and MPS VII mouse (17-19). These studies have provided invaluable information regarding both disease pathogenesis and therapy outcomes.

Concomitant with the desire for improved therapy outcome are the current limitations surrounding monitoring of therapy and the eventual need to tailor therapeutic regimes to individual responses and needs. The advent of ESI-MS/MS has enabled the evaluation of a huge array of compounds present in tissues or body fluids. In MPS, we have identified a number of oligosaccharides derived from the partial degradation of GAG stored in these disorders (20). These oligosaccharides can be quantified in biologic fluids using ESI-MS/MS (21). We have identified a correlation between disease severity and the concentration of these markers in urine and have observed a decrease in concentration after treatment by bone marrow transplantation in MPS IVA and VI (21). As such, these are potentially useful markers to assess the efficacy of therapy in the MPS group of disorders.

ERT in the MPS VI cat has proven very effective in reducing pathology in most connective tissues $(14,16)$. Therapy instituted at birth, before the development of extensive skeletal pathology characteristic of the disease in both humans and cats with MPS VI, significantly reduces the severity of the skeletal abnormalities $(15,16)$. A dose-related response to ERT was observed, with greater overall benefit seen at a $5 \mathrm{mg} / \mathrm{kg}$ (recombinant human 4-sulfatase) dose rate, however avascular connective tissues remained refractory to i.v. ERT $(15,16)$. As soft tissue and skeletal pathology are not fully developed at birth, with significant tissue pathology developing by 1 mo of age (16), the immediate postnatal period is clearly the optimal time for adjusting therapy and modifying the disease process.

In this study, we evaluate the efficacy of ERT in MPS VI cats from birth using an initial high dose of rf4S compared with previous ERT protocols. The aim is to arrest disease progression as early as possible to modify pathology, thereby maximizing the clinical outcome. ESI-MS/MS analysis of blood and urinary oligosaccharides derived from GAG was used to measure dose response as a new method of disease monitoring and compared with established clinical, biochemical, and histologic methods.

\section{METHODS}

Enzyme production and purification. Expression of $\mathrm{rf} 4 \mathrm{~S}$ was performed in Chinese hamster ovary cells and the rf4S was isolated from the culture medium using a MAb affinity column, as described previously (22). Three batches of enzyme were prepared and purity was confirmed by SDS-PAGE under reducing conditions. Protein and activity were determined as described previously (22). Specific activity of the enzyme preparations was $63,530 \pm 10,060 \mathrm{nmol} / \mathrm{min} / \mathrm{mg}$ (mean $\pm \mathrm{SD}$ of $n=3$ preparations). Enzyme dose was calculated according to protein concentration.

Experimental animals. The Women's and Children's Hospital and Institute of Medical and Veterinary Science animal ethics committees approved all studies. All cats used in these studies were bred in an outdoor housed colony originally established from "family 3" heterozygotes (obtained from
Mark Haskins, School of Veterinary Medicine, University of Pennsylvania, Philadelphia, PA, U.S.A.). Newborn kittens were screened for MPS VI by evaluating blood films for characteristic morphologic changes in white blood cells (23). All kittens were subsequently genotyped using PCR-based mutation analysis of blood spots (24).

Enzyme administration and clinical evaluation. Six MPS VI cats were injected intravenously with rf4S on a weekly basis starting within $30 \mathrm{~h}$ of birth. Three animals started at an initial rf4S dose of $20 \mathrm{mg} / \mathrm{kg}$ weekly for the first five doses before the dose was reduced to $1 \mathrm{mg} / \mathrm{kg}$ weekly for the remaining nine doses ("high-dose" treatment group). Three animals were given $1 \mathrm{mg} / \mathrm{kg} \mathrm{rf} 4 \mathrm{~S}$ weekly for all 14 doses ("low-dose" treatment group). A routine clinical examination was performed before the final rf4S infusion then animals were killed $4 \mathrm{~d}$ later at $\sim 90 \mathrm{~d}$ of age by an overdose of anesthetic.

From 6 wk of age, animals were premedicated with $1 \mathrm{mg}$ each of chlorpheniramine (Piriton, GlaxoSmithKline, Uxbridge, Middlesex, U.K.) and cyproheptadine (Periactin, Merck Sharp \& Dohme, South Granville, NSW, Australia) at least 30 min before enzyme administration. At less than $30 \mathrm{~d}$ of age urine samples were collected by perineal massage. Urine from older cats (up to $\sim 90 \mathrm{~d}$ of age) was collected under light sedation or anesthesia as described previously (14). All urine samples were immediately stored at $-20^{\circ} \mathrm{C}$. Whole blood was spotted and dried onto filter paper "Guthrie" cards (S\&S 903, Schleicher \& Schuell, Dassel, Germany). These were collected at weekly intervals from ERT-treated and control cats. Blood was collected immediately before rf4S infusion in ERT animals, which was usually at 7-d intervals, except for the final sample collected at euthanasia $4 \mathrm{~d}$ after the last rf4S infusion. Guthrie cards were stored dried at room temperature for analysis by ESI-MS/MS. Heparinized blood samples for titer determinations were collected 3 and $4 \mathrm{~d}$ after the previous rf4S infusion at 53 and $90 \mathrm{~d}$ of age, respectively, under light sedation or anesthesia. Plasma antibody titers to rf4S were determined using an ELISA method as previously described (22).

Histology. Cats were killed $4 \mathrm{~d}$ after the final rf4S infusion while under general anesthesia, using an overdose of barbiturate. Tissues were collected, fixed, processed routinely as for electron microscopy, and embedded into Spurr's resin (16). One-micrometer-thick resin sections were stained with toluidine blue and were evaluated at $400 \times$ magnification to assess overall distribution of vacuolation due to lysosomal storage. A subjective grading system was used to compare degree of lysosomal vacuolation among different tissues and animals. These grades were as follows: none $(0)$, very mild $( \pm)$, mild $(1+)$, moderate $(2+)$, severe $(3+)$.

Urinary GAG analysis-DMB assay. Quantitation of urinary GAG was performed using a DMB dye-binding assay (25, 26). Briefly, a $10 \times$ stock DMB $(80 \%$, Sigma Chemical, Castle Hill, NSW, Australia) solution $(0.35 \mathrm{mM})$ was made by dissolving $1.2 \mathrm{mg}$ of DMB in $100 \mu \mathrm{L}$ of $95 \%$ ethanol and diluting to $10 \mathrm{~mL}$ with $200 \mathrm{mM} \mathrm{CHOONa}, \mathrm{pH} 3.5$. The stock solution was diluted 1:3 with $200 \mathrm{mM} \mathrm{CHOONa,} \mathrm{pH} \mathrm{3.5,} \mathrm{just} \mathrm{before}$ use. Urine samples $(0.5-5 \mu \mathrm{L})$ were diluted to $50 \mu \mathrm{L}$ with 200 $\mathrm{mM}$ CHOONa, $\mathrm{pH}$ 3.5, in 96-well vinyl microtitre plates 
(Costar, Cambridge, MA, U.S.A.). To each sample, $100 \mu \mathrm{L}$ of the working DMB solution was added, the plate was shaken manually for $2 \mathrm{~s}$, and the OD $520 \mathrm{~nm}$ measured immediately in a Victor2 multilabel reader (PerkinElmer Life Science, Boston, MA, U.S.A.). A calibration curve was generated using chondroitin 6-sulfate from shark cartilage (Sigma Chemical).

Derivatization of oligosaccharides. Samples of urine $(0.5$ $\mu \mathrm{mol}$ creatinine equivalents) were lyophilized before derivatization. Each sample had 2 nmol of GlcNAc6S- $\mathrm{d}_{3}(21)$ and 100 $\mu \mathrm{L}$ of derivatizing solution (250 mM PMP, $400 \mathrm{mM} \mathrm{NH}_{3}, \mathrm{pH}$ 9.1) added. The samples were vortexed vigorously and incubated at $70^{\circ} \mathrm{C}$ for $90 \mathrm{~min}$. Samples were then acidified with a 2 -fold molar excess of formic acid $(100 \mu \mathrm{L}$ of $800 \mathrm{mM}$ solution) and made up to $500 \mu \mathrm{L}$ with water. Each sample was extracted with $500 \mu \mathrm{L}$ of $\mathrm{CHCl}_{3}$ to remove excess PMP and centrifuged $(13,000 \times g, 5 \mathrm{~min})$. Solid phase extraction columns (100 mg, C18 end-capped; International Sorbent Technology, Mid Glamorgan, U.K.) were primed with successive 1 $\mathrm{mL}$ washes of $100 \% \mathrm{CH}_{3} \mathrm{CN}, 50 \% \mathrm{CH}_{3} \mathrm{CN} / 0.025 \%$ formic acid, and water. The aqueous layer from each $\mathrm{CHCl}_{3}$ extraction $(400 \mu \mathrm{L})$ was applied to a primed $\mathrm{C} 18$ column and allowed to enter the solid phase completely. The column was washed with water $(1 \times 500 \mu \mathrm{L}$, followed by $2 \times 1000 \mu \mathrm{L})$ and dried under vacuum (15 min) on a Supelco, Visiprep24 vacuum manifold (Sigma Chemical) or in a lyophilizer (45 min), if in the 96-well format. Each dried $\mathrm{C} 18$ column was then washed with $\mathrm{CHCl}_{3}$ $(2 \times 1000 \mu \mathrm{L})$ to remove any unincorporated PMP, and again dried thoroughly. Derivatized oligosaccharides were eluted from the $\mathrm{C} 18$ column with $50 \% \mathrm{CH}_{3} \mathrm{CN} / 0.025 \%$ formic acid in water $(500 \mu \mathrm{L})$ and injected into the mass spectrometer. Whole blood samples were dried onto filter paper (S\&S 903, Schleicher and Schuell). Three-millimeter punches were taken and derivatized as described above with the following changes: 0.1 nmol GlcNAc6S-d3 was used per sample and $25 \mathrm{mg} \mathrm{C} 18$ columns in a 96-well microtiter format were used. The PMPderivatized samples eluted from the $\mathrm{C} 18$ columns were dried under a stream of $\mathrm{N}_{2}$. Each sample was reconstituted in $100 \mu \mathrm{L}$ of $50 \% \mathrm{CH}_{3} \mathrm{CN} / 0.025 \%$ formic acid in water for injection into the mass spectrometer.

ESI-MS/MS analysis of oligosaccharides. Mass spectrometric analyses were performed in negative ion mode using a PE Sciex API 3000 triple-quadrupole mass spectrometer with a Turboionspray source and Analyst v1.1 data system (MDS Sciex, Concord, ON, Canada ). Samples $(20 \mu \mathrm{L})$ were injected into the electrospray source with a Waters 233XL Autosampler (Waters, Milford, MA, U.S.A.) using a solvent consisting of $50 \% \mathrm{CH}_{3} \mathrm{CN} / 0.025 \%$ formic acid in water, at a flow rate of 80 $\mu \mathrm{L} / \mathrm{min}$. For all analytes, nitrogen was used as both the sheath gas and the collision gas. Quantification of PMP-derivatized oligosaccharides was performed using the multiple-reaction monitoring (MRM) mode. Ion pairs corresponding to sulfated HexNAc and disaccharides containing both HexNAc and UA were monitored as follows: 633/259 (GlcNAc6S- $d_{3}$ ), 630/256 (HexNAcS), 710/256 (HexNAcS 2 ), 806/331 (HexNAcS-UA), and 726/173 (HexNAc-UA and UA-HexNAc). Each ion pair was monitored for $100 \mathrm{~ms}$ using a resolution setting of 0.7 atomic mass units at half peak height for both Q1 and Q3. For each quantitative measurement, continuous scans were made over the injection period and averaged. Quantification was achieved by relating the peak heights of the PMP-oligosaccharides to the peak height of the PMP-GlcNAc6S- $d_{3}$ internal standard using Chemoview v1.0b2 (MDS Sciex, Concord, Ontario, Canada). All oligosaccharides concentrations are reported as relative units.

\section{RESULTS}

Clinical disease progression in MPS VI cats undergoing ERT. Features of MPS VI disease were subjectively milder in the high-dose-treated cats, with cats exhibiting less rounded facial features, larger ears, and a more normal body shape compared with the low-dose-treated cats. All of the low-dosetreated cats had obvious MPS VI disease features, including a broad, rounded face with slightly flattened nose, short neck and tail, broad body shape, and variable but mild degrees of kyphosis of the thoracolumbar spine. One low-dose cat also exhibited very mild hindlimb neurologic deficits consistent with bony compression of the spinal cord as described previously (16).

Histologic and immunologic assessment of therapy. Both enzyme doses resulted in the clearance of storage material from lysosomes in a range of soft connective tissues such as the liver (Table 1). Subjectively, high-dose therapy appeared to give a greater reduction in storage over the low-dose therapy in skin, dura matter, and hip capsule fibroblasts. However, no reduction in storage was observed in articular cartilage or cornea at either dose, with the tissue appearance resembling that of untreated MPS VI tissue. No significant antibody response to rf4S was detected in any MPS VI cats undergoing ERT or in untreated MPS VI and normal control littermates sampled by ELISA (results not shown).

Monitoring of urinary GAG using the DMB assay. Total urine GAG as determined by the DMB assay in the low- and high-dose ERT-treated MPS VI cats gave values midway between untreated MPS VI and control animals (Fig. 1). GAG levels in high-dose-treated cats at $\sim 25 \mathrm{~d}$ of age were nearly

Table 1. Lysosomal storage in various tissues in MPS VI cats after ERT started at birth

\begin{tabular}{|c|c|c|c|}
\hline Tissue/cell type & $\begin{array}{c}\text { No ERT } \\
1 \mathrm{mo}^{*} \\
(n=1-2)\end{array}$ & $\begin{array}{c}1 \mathrm{mg} / \mathrm{kg} \mathrm{rf} 4 \mathrm{~S} \\
(\text { low dose) } \\
3 \mathrm{mo} \\
(n=3)\end{array}$ & $\begin{array}{c}20-1 \mathrm{mg} / \mathrm{kg} \mathrm{rf} 4 \mathrm{~S} \\
\text { (high dose) } \\
3 \mathrm{mo} \\
(n=3)\end{array}$ \\
\hline Liver Kupffer cells & $3+$ & 0 & 0 \\
\hline Brain perivascular cells & $2+$ & $\pm-2+$ & $1+-2+$ \\
\hline Skin fibroblasts & $3+$ & $2+$ & $\pm-2+$ \\
\hline Dura mater fibroblasts & $\mathrm{NA} \S$ & $2+-3+$ & $1+-2+$ \\
\hline Heart valve fibroblasts & $3+$ & $1+-2+$ & $1+-2+$ \\
\hline Aorta smooth muscle cells & $3+$ & $2+$ & $1+-2+$ \\
\hline Hip joint capsule fibroblasts & NA & $1+-3+$ & $2+$ \\
\hline Corneal keratocytes & $3+$ & $3+$ & $3+$ \\
\hline Cartilage chondrocytes & $3+$ & $3+$ & $3+$ \\
\hline
\end{tabular}

I Degree of lysosomal vacuolation is graded as none $(0)$, very mild $( \pm)$, mild $(1+)$, moderate $(2+)$, severe $(3+)$. Grade is 0 for all tissues at all ages in normal cats.

$\S$ Not available.

* From Crawley et al. 16. Lysosomal storage is maximal by 1 mo of age in all of the tissues examined above, except brain perivascular cells, which achieve maximal levels within 6 mo. 


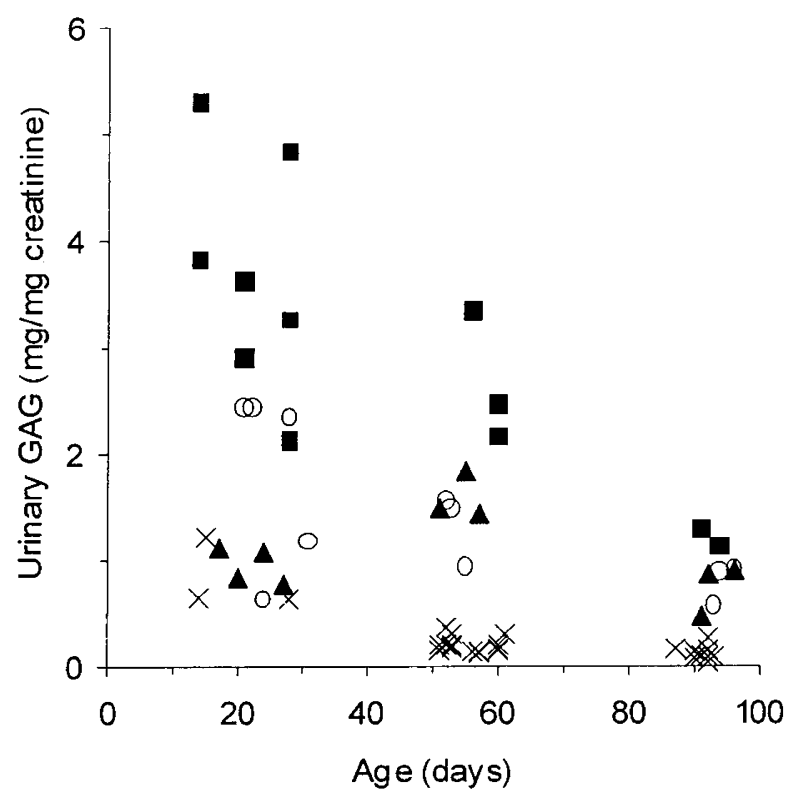

Figure 1. Total urinary GAG from control, treated and untreated animals. Urine was collected from normal $(\times)$, MPS VI untreated ( $\square$ ), MPS VI low-dose-treated $(\bigcirc)$, and MPS VI high-dose treated $(\mathbf{\Delta})$ animals at the times indicated. Total urinary GAG was determined using the DMB dye binding assay and normalized to creatinine.

normalized, compared with the low-dose-treated cats, consistent with the higher rf $4 \mathrm{~S}$ dose rate administered in the first $28 \mathrm{~d}$ of age. At days $\sim 55$ and 90 , the level of urinary GAG was similar in both the high- and low-dose animals.

Monitoring of urinary GAG-derived oligosaccharides using $\boldsymbol{E S I - M S / M S}$. Two oligosaccharides previously shown to correlate to disease state in human MPS VI (21) were analyzed in normal, MPS VI-affected, and enzyme-treated MPS VI cats. Significant levels of the monosaccharide HexNAcS was detected in urine from untreated MPS VI cats. Levels were 4- to 5 -fold higher than in normal animals at $15-25 \mathrm{~d}$ of age and seven times the levels found in normal cats at $90 \mathrm{~d}$ (Fig. 2A). Initial monosaccharide sulfate concentrations at $25 \mathrm{~d}$ in the low-dose-treated group were similar to untreated MPS VI cats. The high-dose-treated group in contrast was much closer to normal levels. However by $\sim 55 \mathrm{~d}$ of age (where both groups were on the $1 \mathrm{mg} / \mathrm{kg}$ dosage), monosaccharide sulfate levels in both ERT dosage groups were similar to each other and approaching levels mid-range between the normal and untreated MPS VI cats. A similar excretion pattern is observed for the sulfated disaccharide HexNAcS-UA, which was $\sim 15$ times the level observed in normal cats at $15-25 \mathrm{~d}$ and $\sim 37$ times the level observed at $90 \mathrm{~d}$ (Fig. 2B). Commensurate with the monosaccharide sulfate results, the initial amounts of sulfated disaccharide in urine of animals in the high-dose-treated group were closer to normal values whereas sulfated disaccharide amounts in urine of the low-dose animals were closer to the untreated MPS VI animals. By $\sim 55 \mathrm{~d}$ of age, sulfated disaccharide amounts in the urine of both ERT dosage groups were similar to each other.

The unsulfated disaccharide HexNAc-UA, represented by the 726/173 MRM pair, is a nonstorage GAG-derived analyte that is independent of MPS VI disease state and provides an
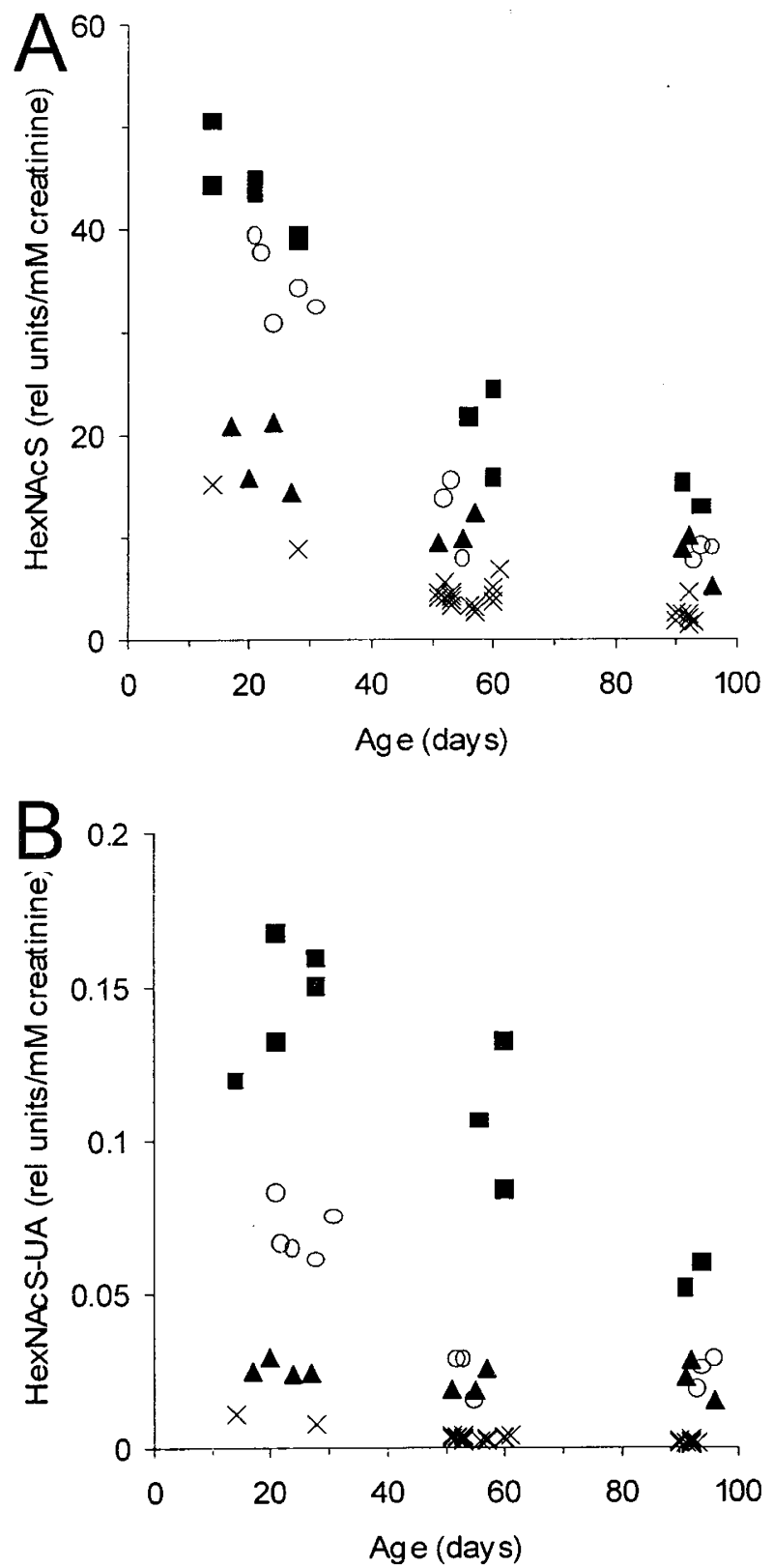

Figure 2. Urinary levels of the monosaccharide (HexNAcS) and the disaccharide (HexNAcS-UA) in control, treated, and untreated animals. Urine was collected from normal $(\times)$, MPS VI untreated $(\boldsymbol{\square})$, MPS VI low-dose-treated $(\bigcirc)$, and MPS VI high-dose-treated $(\boldsymbol{\Delta})$ animals at the times indicated. The relative concentrations of the monosaccharide HexNAcS $(A)$ and the disaccharide HexNAcS-UA $(B)$ were measured using tandem mass spectrometry and normalized to creatinine.

alternative normative measure to creatinine. When the amount of the sulfated disaccharide HexNAcS-UA is plotted as a ratio of HexNAc-UA, a different pattern is observed over time (Fig. 3). The ratio of HexNAcS-UA to HexNAc-UA did not decrease with age and instead displayed a slight increase. HexNAcS-UA to HexNAc-UA ratios were $\sim 33$ times the normal cat value at $21 \mathrm{~d}$ compared with 47 times the normal cat value at $90 \mathrm{~d}$. At the early time point, a difference was noted between animals in the different ERT groups. High-dose animals had urinary HexNAcS-UA:HexNAc-UA values close to normal, whereas low-dose animals were comparable to untreated MPS 


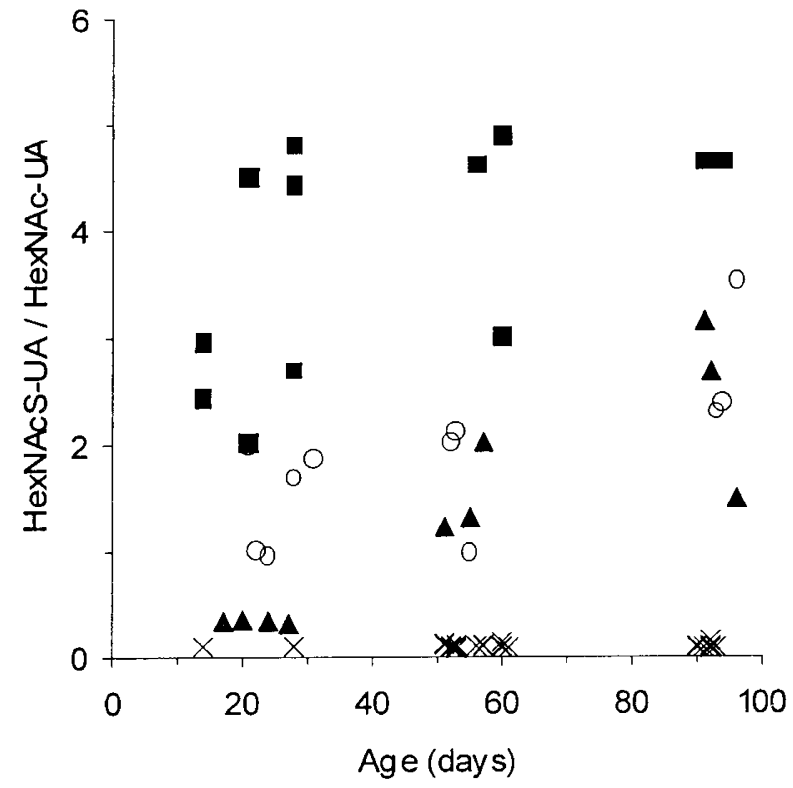

Figure 3. Urinary ratio of the monosaccharide to disaccharide (HexNAcS/ HexNAcS-UA) in control, treated, and untreated animals. Urine was collected from normal $(\times)$, MPS VI untreated $(\square)$, MPS VI low-dose-treated $(\bigcirc)$, and MPS VI high-dose-treated $(\mathbf{\Delta})$ animals at the times indicated. The relative concentrations of the disaccharides HexNAcS-UA and HexNAc-UA were measured using tandem mass spectrometry and are expressed as a ratio.

VI animals. As with previous results, by $\sim 55 \mathrm{~d}$ of therapy the urinary HexNAcS-UA:HexNAc-UA values were similar in both treatment groups. In contrast to previous results, normalized to creatinine, the ratio of HexNAcS-UA to HexNAc-UA in the urine of all animals increased with age.

Statistical analysis of the urine data grouped as $10-30 \mathrm{~d}$, $50-70 \mathrm{~d}$, and $80-100 \mathrm{~d}$, using independent samples $t$ test was performed. This analysis showed that determination of total urinary GAG could not discriminate the high- and low-dose groups at $10-30 \mathrm{~d}(p=0.09)$, whereas the oligosaccharide markers and ratios were able to discriminate these groups $(p<$ 0.01 ). Similarly, at $80-100 \mathrm{~d}$ neither of the treated groups could be distinguished from the untreated group using total urinary GAG $(p>0.05)$ but could be distinguished using the oligosaccharide markers and ratios $(p<0.05)$.

Monitoring of GAG-derived oligosaccharides in blood. HexNAcS concentration in the untreated MPS VI group displayed an overall increase with age whereas the control cats maintained a consistently low level (Fig. 4). Low- and highdose ERT cats display dose-related levels of HexNAcS in blood. Higher initial levels can be seen in the low-dose group compared with the high-dose group. Both groups then plateaued approximately midway between the untreated MPS VI and normal cats by $90 \mathrm{~d}$ of age. A similar pattern in analyte levels was observed between normal, MPS VI-, and MPS VI-treated animals in the circulation as observed in the urine (Fig. 4 compared with Fig. 2B). However, the discrimination between treatment groups was not as clear as that observed in urine.

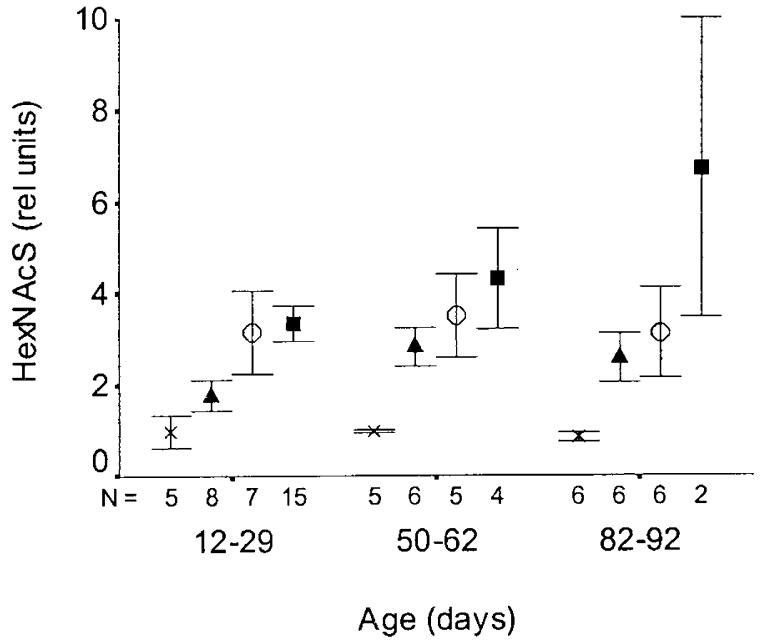

Figure 4. Concentration of the monosaccharide (HexNAcS) in blood from control, treated, and untreated animals. Blood was collected from normal $(X)$, MPS VI untreated (ם), MPS VI low-dose-treated $(\bigcirc)$, and MPS VI high-dosetreated $(\boldsymbol{\Delta})$ animals. The concentration of the monosaccharide HexNAcS was determined using tandem mass spectrometry. Values are presented as the mean $\pm 2 \mathrm{SE}$ for the age range indicated. The number of data points per age range is indicated below the $x$ axis.

\section{DISCUSSION}

ESI-MSMS enables the determination of a vast array of compounds and metabolites in biologic systems. This raises the prospect of biochemical monitoring of disease progression and therapeutic outcomes, once appropriate biochemical markers are identified for individual diseases. With the likelihood of ERT becoming available for a number of LSD, including MPS VI, methods for monitoring the response to therapy will be required to tailor individual therapeutic regimes and thereby optimize clinical outcomes.

ERT, as evaluated in feline MPS VI, has proven effective in reducing the lysosomal storage in soft connective and skeletal tissues in a dose-dependent manner (14-16). Treated animals attain a more normal appearance and display a freer range of movement than untreated animals. Some tissues such as cartilage and cornea, however, remain unaffected by ERT (16). Analysis of the natural progression of pathology in the MPS VI cat has shown that the degree of lysosomal vacuolation increases with age, becoming maximal approximately 1 mo after birth. The birth to 1-mo period presents a time frame in which it might be possible to alter the clinical progression of MPS VI by the modulation of ERT during the period before pathology becomes entrenched.

In this study, the administration of a very high dose of rf4S in the immediate postnatal period was monitored by the determination of the GAG-derived oligosaccharides HexNAcS and HexNAcS-UA in urine. These biochemical markers showed a dose response during the first 4 wk of treatment, when ERT dose rates were different between the two treated groups of animals. Both analytes, in particular the sulfated disaccharide HexNAcS-UA, provided a clearer discrimination between the treatment groups than total GAG determination by the commonly used DMB method (compare Fig. 1 and Fig. 2B). 
Conversely, the initial high-dose regimen did not confer any significant advantage over the standard dosing regimen with respect to clearance of lysosomal storage from cartilage and cornea, both avascular connective tissues, despite subjective differences in overall physical appearance. However, both dose rates did remove or prevent accumulation of lysosomal storage in a number of soft connective tissues, compared with untreated MPS VI cats. Subjective improvements in the initial high-dose group compared with the low-dose group were observed in skin, dura matter, and hip capsule fibroblasts, and in aorta smooth muscle cells in some animals.

The analytes evaluated by ESI-MSMS (HexNAcS and HexNAcS-UA) are presumed to be GalNAc4S and GalNAc4S-UA, respectively, because of the known deficiency of $N$-acetylgalactosamine-4-sulfatase in MPS VI. The origins of the analytes are thought to arise from two different mechanisms of GAG catabolism. The monosaccharide sulfate GalNAc4S on the nonreducing end of the dermatan sulfate storage product can be cleaved from the intact GAG by an alternative but inefficient pathway involving an exo-enzyme; $\beta$ - $N$-acetylhexosaminidase $(27,28)$. The GalNAc4S-UA disaccharide, however, must result from another, as yet uncharacterized, degradation pathway involving an endoenzyme in combination with the exo-enzymes involved in GAG degradation. The endo-lytic digestion of GAG would be expected to generate a range of fragments of intermediate size. These would then be trimmed back to structures having a 4-sulfated $\mathrm{N}$ acetylgalactosamine moiety on the nonreducing terminal by the action of the lysosomal exo-enzymes. A potential candidate for the endo-enzyme is the hyaluronidase family of endoglycosidases, which are known to cleave hyaluronan, chondroitin sulfate, and, to a lesser extent, dermatan sulfate GAG (29). At least one member of the family is found in the lysosome (30).

An advantage of tandem mass spectrometry determination of urinary oligosaccharides is the ability to use other nondiseaserelated oligosaccharides as the normative value instead of creatinine. Although commonly used as a measure of GFR, urinary creatinine values correlate to muscle mass and, as such, increase with age as body weight accumulates (31). As a result, urinary GAG levels decrease with age when normalized to creatinine. The unsulfated disaccharide HexNAc-UA (and/or UA-HexNAc) represented by the 726/173 MRM pair, which was not elevated in MPS VI urine (data not shown), was used to normalize the previously identified sulfated disaccharide (Fig. 3). The ratio of HexNAcS-UA to HexNAc-UA/UA-HexNAc shows a constant low value in the control animals, effectively correcting for the decrease in urinary GAG excretion observed with age. The highdose animals displayed almost normalized values at $15-25 \mathrm{~d}$, but these increased when the animals reverted to the low-dose regimen and were indistinguishable from the low-dose animals at $50 \mathrm{~d}$. This suggests an increase in GAG storage and subsequent urinary excretion during low-dose therapy compared with the high-dose and is further supported by the continuous increase in the ratio value in the low-dose animals over the $90 \mathrm{~d}$ of therapy. The increase in the ratio value in both groups of treated animals, however, occurred at a slower rate than the untreated MPS VI animals and did not attain the same level. These results were not evident from the total urinary GAG determination (Fig. 1) and demonstrate the advantages of these markers for the monitoring of therapy for MPS VI.

The determination of oligosaccharides in blood collected as dried blood spots is an attractive proposition for the monitoring of therapy due to the ease of collection and the convenience of storage and transport. In this study, blood was collected from treated and untreated animals at the same time as urine collection to compare oligosaccharide levels in each fluid. Only the sulfated monosaccharide HexNAcS was present in dried blood spots in sufficient concentrations to provide useful data. The level of this analyte in blood showed a similar trend to that seen in the corresponding urine samples, although there was considerable variation in both the high and low treatment groups, possibly due to a circadian fluctuation in circulating GAG levels. Further analysis is required to optimize ESI-MS/MS measurement of disease-related oligosaccharides in blood.

In summary, the administration of transient high-dose ERT in the immediate postnatal period cannot be justified in the absence of a significant improvement in lysosomal storage in the severely affected avascular connective tissues. The potential for increased side-effects arising from the administration of high protein amounts and the increased costs associated with production of large amounts of recombinant enzyme would offset the small improvements observed. The use of ESI-MS/MS to monitor the urinary excretion of oligosaccharides representative of MPS VI disease state has a number of potential advantages. Firstly, it provides a noninvasive method to monitor GAG load during therapy, as opposed to histologic studies. Secondly, it has the ability to monitor urinary GAG levels at independent time points during therapy and removes the need to normalize to creatinine, whereas the standard DMB or Alcian blue method requires all samples to be analyzed simultaneously and corrected for creatinine to provide a valid comparison. The use of ESI-MS/MS would be applicable to other MPS in which a urinary marker of disease can be identified, and work is continuing in this area. Further studies will demonstrate the robustness of ESI-MS/MS as a technique for both the diagnosis of MPS and to monitor therapy regimens to tailor treatment to the individual.

Acknowledgments. The authors thank Mark Haskins, University of Pennsylvania, for provision of the original MPS VI heterozygote cats. We also thank the animal care staff at the Institute of Medial and Veterinary Science, Adelaide for care and maintenance of the cat colony; Peter Gray and Jodie Varnai for preparation of culture medium containing rf4S; Kaye Beckman for purification of rf4S; and Tina Rozaklis for the collation of samples for MSMS analysis.

\section{REFERENCES}

1. Hopwood JJ, Brooks DA 1997 An introduction to the basic science and biology of the lysosome and storage diseases. In: Applegarth DA, Dimmick JE, Hall JG (eds) Organelle Diseases. Chapman and Hall Medical, London, pp 7-36

2. Meikle PJ, Hopwood JJ, Clague AE, Carey WF 1999 Prevalence of lysosomal storage disorders. JAMA 281:249-254

3. Barton NW, Brady RO, Dambrosia JM, Di Bisceglie AM, Doppelt SH, Hill SC, Mankin HJ, Murray GJ, Parker RI, Argoff CE 1991 Replacement therapy for inherited enzyme deficiency - macrophage-targeted glucocerebrosidase for Gaucher's disease. N Engl J Med 324:1464-1470

4. Eng CM, Guffon N, Wilcox WR, Germain DP, Lee P, Waldek S, Caplan L, Linthorst GE, Desnick RJ 2001 Safety and efficacy of recombinant human alpha-galactosidase A-replacement therapy in Fabry's disease. N Engl J Med 345:9-16 
5. Kakkis ED, Muenzer J, Tiller GE, Waber L, Belmont J, Passage M, Izykowski B, Phillips J, Doroshow R, Walot I, Hoft R, Neufeld EF 2001 Enzyme-replacement therapy in mucopolysaccharidosis I. N Engl J Med 344:182-188

6. Weinreb NJ, Charrow J, Andersson HC, Kaplan P, Kolodny EH, Mistry P, Pastores G, Rosenbloom BE, Scott CR, Wappner RS, Zimran A 2002 Effectiveness of enzyme replacement therapy in 1028 patients with type 1 Gaucher disease after 2 to 5 years of treatment: a report from the Gaucher Registry. Am J Med 113:112-119

7. Van den Hout H, Reuser AJ, Vulto AG, Loonen MC, Cromme-Dijkhuis A, Van de Ploeg AT 2000 Recombinant human alpha-glucosidase from rabbit milk in Pompe patients. Lancet 356:397-398

8. Harmatz P, Whitley CB, Belani K, Waber L, Pais R, Steiner R, Plecko B, Simon J, Thompson J, Waterson J, Lammer E, Rowe R, Koseglu S, Cohen R, Rosenfeld H, Gutierrez H, Hopwood JJ 2001 A phase I/II randomised, double blind, two dose group study of recombinant human N-acetylgalactosamine-4-sulfatase (rhASB) enzyme replacement therapy in patients with mucopolysaccharidosis (MPS) VI (MaroteauxLamy syndrome). Am J Hum Genet 69(suppl 4):2896

9. Cabrera-Salazar MA, Novelli E, Barranger JA 2002 Gene therapy for the lysosomal storage disorders. Curr Opin Mol Ther 4:349-358

10. Desnick RJ, Schuchman EH 2002 Enzyme replacement and enhancement therapies: lessons from lysosomal disorders. Nat Rev Genet 3:954-966

11. Shull RM, Kakkis ED, McEntee MF, Kania SA, Jonas AJ, Neufeld EF 1994 Enzyme replacement in a canine model of Hurler syndrome. Proc Natl Acad Sci U S A 91:12937-12941

12. Kakkis ED, McEntee MF, Schmidtchen A, Neufeld EF, Ward DA, Gompf RE, Kania S, Bedolla C, Chien SL, Shull RM 1996 Long-term and high-dose trials of enzyme replacement therapy in the canine model of mucopolysaccharidosis I. Biochem Mol Med 58:156-167

13. Kakkis ED, Schuchman E, He X, Wan Q, Kania S, Wiemelt S, Hasson CW, O’Malley T, Weil MA, Aguirre GA, Brown DE, Haskins ME 2001 Enzyme replacement therapy in feline mucopolysaccharidosis I. Mol Genet Metab 72:199-208

14. Crawley AC, Brooks DA, Muller VJ, Petersen BA, Isaac EL, Bielicki J, King BM, Boulter CD, Moore AJ, Fazzalari NL, Anson DS, Byers S, Hopwood JJ 1996 Enzyme replacement therapy in a feline model of Maroteaux-Lamy syndrome. J Clin Invest 97:1864-1873

15. Byers S, Nuttall JD, Crawley AC, Hopwood JJ, Smith K, Fazzalari NL 1997 Effect of enzyme replacement therapy on bone formation in a feline model of mucopolysaccharidosis type VI. Bone 21:425-431

16. Crawley AC, Niedzielski KH, Isaac EL, Davey RC, Byers S, Hopwood JJ 1997 Enzyme replacement therapy from birth in a feline model of mucopolysaccharidosis type VI. J Clin Invest 99:651-662

17. Vogler C, Sands M, Higgins A, Levy B, Grubb J, Birkenmeier EH, Sly WS 1993 Enzyme replacement with recombinant beta-glucuronidase in the newborn mucopolysaccharidosis type VII mouse. Pediatr Res 34:837-840
18. Sands MS, Vogler C, Kyle JW, Grubb JH, Levy B, Galvin N, Sly WS, Birkenmeier EH 1994 Enzyme replacement therapy for murine mucopolysaccharidosis type VII. J Clin Invest 93:2324-2331

19. Vogler C, Sands MS, Levy B, Galvin N, Birkenmeier EH, Sly WS 1996 Enzyme replacement with recombinant beta-glucuronidase in murine mucopolysaccharidosis type VII: impact of therapy during the first six weeks of life on subsequent lysosomal storage, growth, and survival. Pediatr Res 39:1050-1054

20. Byers S, Rozaklis T, Brumfield LK, Ranieri E, Hopwood JJ 1998 Glycosaminoglycan accumulation and excretion in the mucopolysaccharidoses: characterization and basis of a diagnostic test for MPS. Mol Genet Metab 65:282-290

21. Ramsay SL, Meikle PJ, Hopwood JJ 2003 Determination of monosaccharides and disaccharides in mucopolysaccharidoses patients by electrospray ionisation mass spectrometry. Mol Genet Metab 78:193-204

22. Bielicki J, Crawley AC, Davey RC, Varnai JC, Hopwood JJ 1999 Advantages of using same species enzyme for replacement therapy in a feline model of mucopolysaccharidosis type VI. J Biol Chem 274:36335-36343

23. Crawley AC, Yogalingam G, Muller VJ, Hopwood JJ 1998 Two mutations within feline mucopolysaccharidosis type VI colony cause three different clinical phenotypes. J Clin Invest 101:109-119

24. Yogalingam G, Litjens T, Bielicki J, Crawley AC, Muller V, Anson DS, Hopwood JJ 1996 Feline mucopolysaccharidosis type VI. Characterization of recombinant Nacetylgalactosamine 4-sulfatase and identification of a mutation causing the disease. J Biol Chem 271:27259-27265

25. Whitley CB, Ridnour MD, Draper KA, Dutton CM, Neglia JP 1989 Diagnostic tes for mucopolysaccharidosis. I. Direct method for quantifying excessive urinary glycosaminoglycan excretion. Clin Chem 35:374-379

26. Whitley CB, Spielmann RC, Herro G, Teragawa SS 2002 Urinary glycosaminoglycan excretion quantified by an automated semimicro method in specimens conveniently transported from around the globe. Mol Genet Metab 75:56-64

27. Hopwood JJ, Elliott $\mathrm{H} 1983$ Isolation and characterization of $N$-acetylglucosamine 6-sulfate from the urine of a patient with Sanfilippo type D syndrome and its occurrence in normal urine. Biochem Int 6:831-836

28. Hopwood JJ, Elliott H 1985 Urinary excretion of sulphated $N$-acetylhexosamines in patients with various mucopolysaccharidoses. Biochem J 229:579-586

29. Frost GI, Csoka T, Stern R 1996 The hyaluronidases: a chemical, biological and clinical overview. Trends Glycosci Glycotechnol 8:419-434

30. Triggs-Raine B, Salo TJ, Zhang H, Wicklow BA, Natowicz MR 1999 Mutations in HYAL1, a member of a tandemly distributed multigene family encoding disparate hyaluronidase activities, cause a newly described lysosomal disorder, mucopolysaccharidosis IX. Proc Natl Acad Sci U S A 96:6296-6300

31. Yared A, Ichikawa I 1994 Pediatric Nephrology, 3rd Ed. Williams and Wilkins, Baltimore, pp 74 